\title{
Espécies novas de Chromatonotus Hebard, 1920 da região da Amazônia (Blattellidae, Blattellinae)
}

\author{
Sonia Maria Lopes ${ }^{1,2}$ \& Andréa Khouri ${ }^{1}$
}

${ }^{1}$ Departamento de Entomologia, Museu Nacional, Universidade Federal do Rio de Janeiro, Quinta da Boa Vista, São Cristóvão, CEP 20940-040, Rio de Janeiro, RJ, Brasil

${ }^{2}$ Autor para correspondencia: Sonia Maria Lopes, e-mail: sonialf@acd.ufrj.br

LOPES, S.M. \& KHOURI, A. 2008. New species of Chromatonotus Hebard, 1920 from the Amazon region (Blattellidae, Blattellinae). Biota Neotrop. 8:(2): http://www.biotaneotropica.org.br/v8n2/en/ abstract?article+bn02308022008.

Abstract: Two new species of Chromatonotus Hebard, 1920 from Mato Grosso State, Brazil, are described: Chromatonotus chirostylatus sp. nov and Chromatonotus sinopensis sp. nov. Illustrations of the shape and coloration, tergal modification in the abdome, genital plates and the male genitalia are presented.

Keywords: description, new species, Blattaria, taxonomy, Amazon.

Lopes, S.M. \& Khouri, A. 2008. Espécies novas de Chromatonotus Hebard, 1920 da região da Amazônia (Blattellidae, Blattellinae). Biota Neotrop. 8:(2): http://www.biotaneotropica.org.br/v8n2/pt/ abstract?article+bn02308022008.

Resumo: Duas espécies novas de Chromatonotus Hebard, 1920 do estado de Mato Grosso, Brasil, são descritas: Chromatonotus chirostylatus sp. nov e Chromatonotus sinopensis sp. nov. Ilustrações sobre a forma e coloração, modificação tergal no abdome, placas genitais e peças da genitália do macho são apresentadas.

Palavras-chave: descrição, nova espécie, Blattaria, taxonomia, Amazônia. 


\section{Introdução}

Chromatonotus foi descrito por Hebard (1920) que designou C. lamprus, coletado no Panamá, como espécie-tipo. O gênero apresenta, até o momento, onze espécies descritas que se distribuem desde a Guatemala até o Brasil na região amazônica brasileira. O gênero Chromatonotus caracteriza-se por apresentar tamanho pequeno, entre 9-15 mm e coloração castanha-amarelada. Na cabeça, o espaço interocular é amplo, pouco menor que o espaço entre as bases das antenas; ocelos são distintos e o vértice é pouco exposto. $\mathrm{O}$ pronoto tem a forma subtrapezoidal com as abas laterais arredondadas e defletidas. Em ambos os sexos as tégminas e asas membranosas são longas e ultrapassam o ápice dos cercos; essas com campo anal dobrado em leque e triângulo apical desenvolvido. As pernas são espinhosas. $\mathrm{Na}$ face ântero-ventral, no fêmur anterior, há de quatro a seis espinhos fortes, da base até a região mediana e uma série de pequenos espinhos até o ápice e três espinhos apicais robustos. Os pulvilos estão presentes em todos os artículos tarsais e as unhas são simétricas e simples com arólios presentes. No abdome há ausência de modificação tergal nos sétimo e oitavo segmentos (Hebard 1920).

Neste trabalho é apresentado um novo registro no Brasil para o gênero, no Estado do Mato Grosso, é assinalado mais um caráter morfológico para melhor identificação entre os demais gêneros e são descritas duas espécies novas de Chromatonotus: C. chirostylatus sp. nov. e $C$. sinopensis sp. nov.

\section{Material e Métodos}

O material foi coletado no município de Sinop, ao longo da BR-163, que atravessa uma série de paisagens características do estado de Mato Grosso. O estado do Mato Grosso pode ser subdividido em duas bacias hidrográficas (Prata e Amazônica) com diferentes biomas, no caso Cerrado, Mata Amazônica e Pantanal. Porém, todo o norte desse estado está no domínio amazônico. Desde 1953, o município de Sinop pertence à região denominada Amazônia Legal, juntamente com os estados do Acre, Amapá, Amazonas, Mato Grosso, Pará, Rondônia, Roraima, Tocantins, e parte do Maranhão (a oeste do meridiano de $44^{\circ} \mathrm{WGr}$.). Tal região perfaz aproximadamente $5.217 .423 \mathrm{~km}^{2}$, o que corresponde a cerca de $60 \%$ do território brasileiro (AmbienteBrasil 2000-2008).

Em laboratório, a observação das placas genitais foi feita através da retirada da parte final do abdome, utilizando-se as técnicas tradicionais de dissecção, descritas em Lopes \& Oliveira (2000). Após análise, as placas e peças genitais foram guardadas em microtubos contendo glicerina, acondicionados junto ao exemplar respectivo, técnica desenvolvida por Gurney et al. (1964). A terminologia da genitália e a classificação taxonômica foram baseadas nos conceitos propostos por Roth (2003). Para identificação precisa, o material foi comparado com os exemplares de Chromatonotus da coleção de Blattaria do Museu Nacional (MNRJ) e com as descrições em literatura. Os exemplares descritos neste trabalho encontram-se depositados na coleção do Departamento de Entomologia do Museu Nacional do Rio de Janeiro (MNRJ).

\section{Resultados}

\section{Chromatonotus chirostylatus sp. nov}

Coloração geral: Castanha hialina e brilhante (Figura 1a); cabeça na base das antenas com pequenas manchas triangulares voltadas para a fronte (Figura 2a), primeiros e segundos artículos dos palpos maxilares castanho-claros, quarto e quinto com tomentosidade dourada; pronoto com uma faixa transversal castanho-
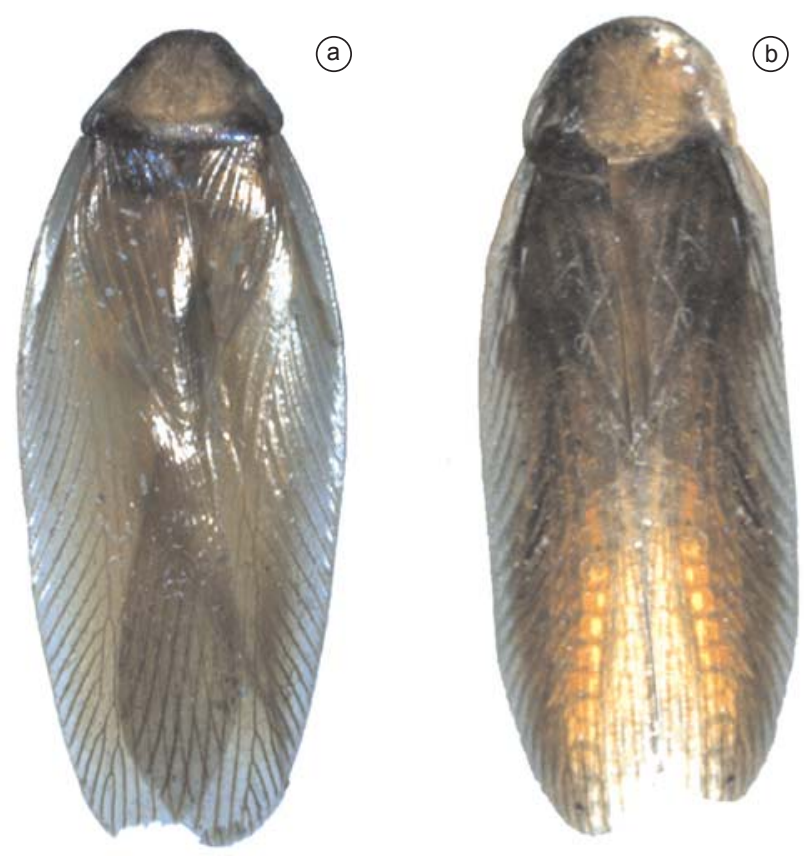

Figura 1. Habitus. a) Chromatonotus chirostylatus sp. nov. Holótipo $\widehat{\delta}$ (13 mm); e b) Chromatonotus sinopensis sp. nov. Holotipo ठ̊ (10 mm).

Figure 1. Habitus. a. Chromatonotus chirostylatus sp. nov. Holotype $\hat{\sigma}$ $(13 \mathrm{~mm})$; and b) Chromatonotus sinopensis sp. nov. Holotype $\widehat{\jmath}(10 \mathrm{~mm})$.

escura na base com manchas delgadas e encurvadas, de mesma coloração em cada uma das regiões látero-apicais (Figura 2b).

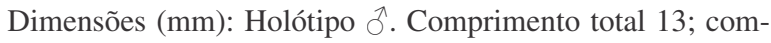
primento do pronoto 2 ; largura do pronoto 3,5 ; comprimento da tégmina 11; largura da tégmina 3.

Abdome: Primeiro tergito com dois conjuntos simétricos de cerdas próximos à região mediana. Placa supra-anal ciliada transversa, com bordo apical ciliado e levemente projetado com suave reentrância (Figura 2c). Cercos com 11 artículos. Placa subgenital assimétrica, ciliada no terço apical, alargada com invaginação acentuada médio-apicalmente onde se inserem os estilos, assimétricos, o esquerdo apicalmente em forma de uma mão e o direito simples e ciliado (Figura 2d). Genitália com esclerito mediano afilado (Figura $2 \mathrm{~g}$ ), falômero esquerdo em forma de gancho simples (Figura 2f); falômero direito em forma de " $Y$ " invertido, com os braços desenvolvidos diferenciados e estrutura mediana laminar esclerotinizada (Figura 2e).

Material examinado: Holótipo $\widehat{o}^{\lambda}$. Brasil, Mato Grosso, Sinop, BR-163, km 500-600, $350 \mathrm{~m}, 12^{\circ} 31^{\prime} \mathrm{S}$ and 55 $27^{\circ} \mathrm{W}$, Alvarenga \& Roppa cols., IX/1974; Parátipo $\widehat{\partial}$, dados de coleta, data e coletor iguais ao Holótipo (MNRJ).

Etimologia: Do grego "cheiros", significando mão, devendo-se à configuração do estilo esquerdo em forma de uma mão.

Comentário: A espécie é similar a $C$. infuscatus (Brunner, 1906) pelo posicionamento dos estilos na região médio-apical da placa subgenital, no entanto, diferindo dessa e das demais espécies do gênero pela configuração da referida placa e das estruturas genitais.

\section{Chromatonotus sinopensis sp. nov.}

Coloração geral: Castanho-clara e brilhante (Figura 1b); cabeça com manchas entre as antenas, na base dos olhos, no clípeo e na lateral do labro (Figura 3a); disco central do pronoto com uma 


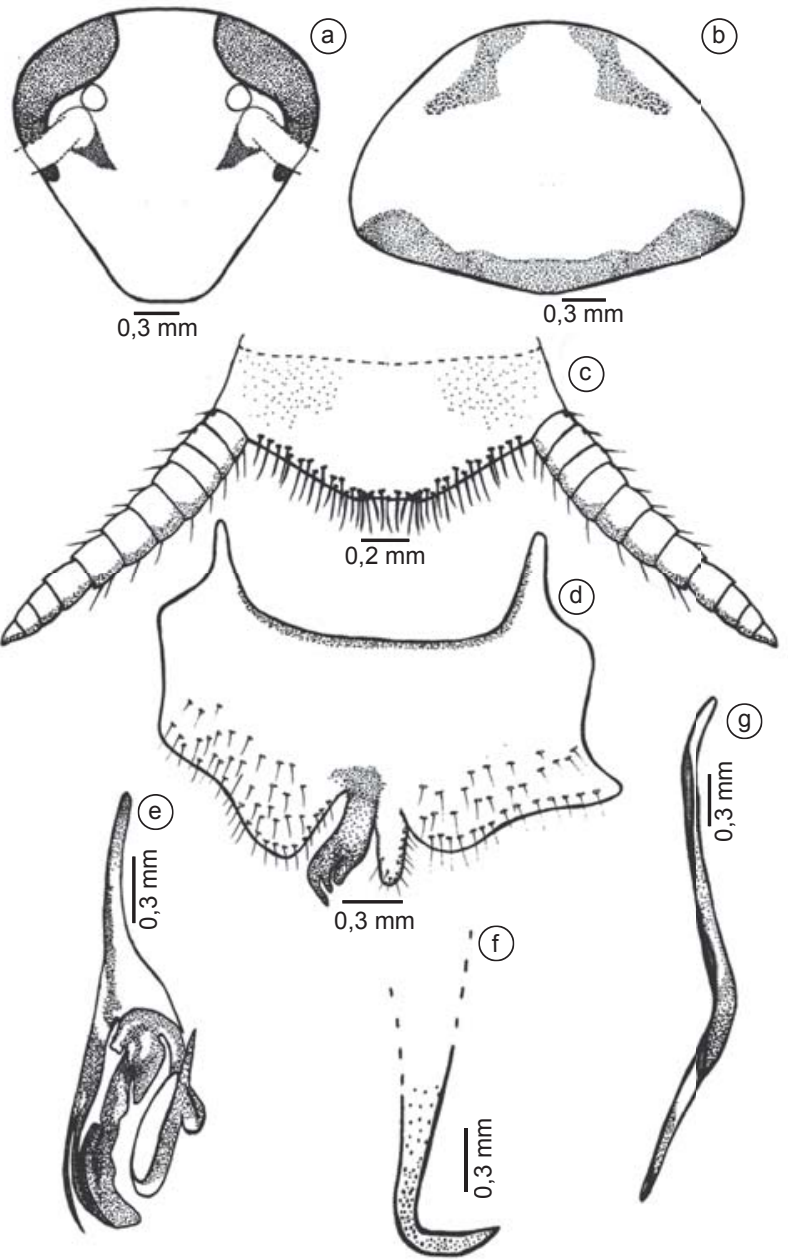

Figura 2. Chromatonotus chirostylatus sp. nov. Holótipo $\hat{\partial}$ a) cabeça; b) pronoto; c) placa supra-anal; d) placa subgenital; e) falômero direito; e f) falômero esquerdo; g. esclerito mediano.

Figure 2. Chromatonotus chirostylatus sp. nov. Holotype ôे a) head; b) pronotum; c) supra-anal plate; d) subgenital plate; e) right phallomere; f) left phallomere; g) median sclerite.

mancha castanho-escura semelhante a um "C" em cada lateral e uma faixa basal irregular de mesma coloração (Figura 3b); quarto e quinto artículos dos palpos maxilares tomentosos castanho-escuros.

Dimensões (mm): Holótipo $\widehat{\partial}$. Comprimento total 10; comprimento do pronoto 2,5 ; largura do pronoto 3 ; comprimento da tégmina 8,5; largura da tégmina 2.

Abdome: Primeiro tergito com dois conjuntos simétricos de cerdas, pouco visíveis, próximo à região mediana do segmento. Placa supra-anal modificada látero-apicalmente, fracamente transversa, margens livres, retas, arredondadas com emarginação mediana no bordo apical e leve reentrância mediana apical. Cercos curtos com cerca de oito a dez artículos ciliados e bem demarcados (Figura 3c). Placa subgenital levemente assimétrica ciliada na metade apical, estilos assimétricos e com uma proeminência entre eles, o direito um pouco mais visível que o esquerdo (Figura 3d). Genitália com esclerito mediano estiliforme e afilado apicalmente (Figura 3e). Falômero direito em forma de "Y" invertido com um dos braços afilado, desenvolvido e com

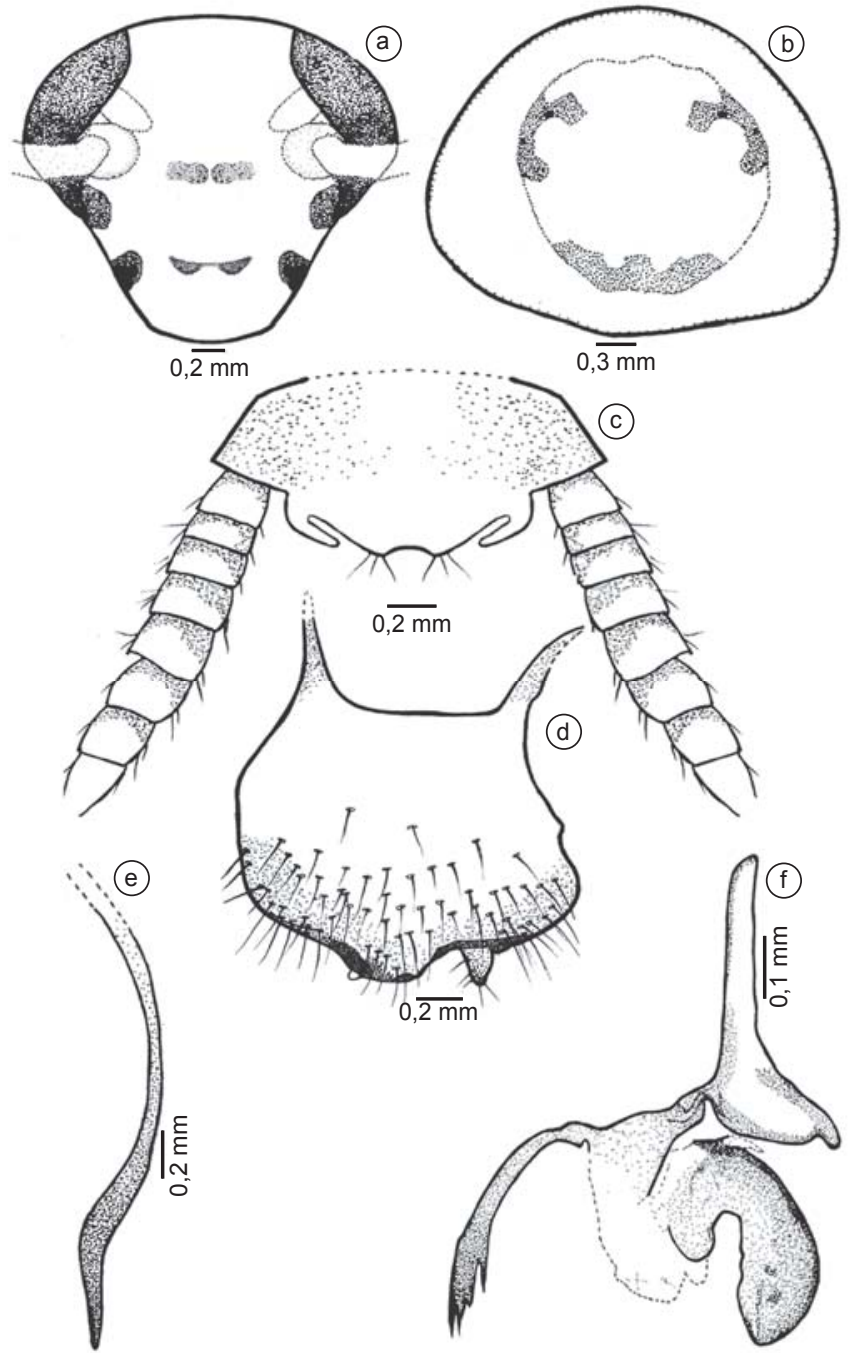

Figura 3. Chromatonotus sinopensis sp. nov. Holótipo đ̋̃ a. cabeça; b) pronoto; c) placa supra-anal; d) placa subgenital; e) esclerito mediano; and f) falômero direito.

Figure 3. Chromatonotus sinopensis sp. nov. Holotype $\widehat{\partial}$ a) head; b) pronotum; c) supra-anal plate; d) subgenital plate; e) median sclerit; and f) right phallomere.

espinhos apicais e o outro um pouco menor e alargado e estrutura mediana laminar (Figura 3f).

Material examinado: Holótipo $\widehat{\jmath}$. Brasil, Mato Grosso, Sinop, BR-163, km 500-600, $350 \mathrm{~m}, 12^{\circ} 31^{\prime} \mathrm{S}$ and $55^{\circ} 27^{\prime} \mathrm{W}$, Alvarenga \& Roppa cols., IX/1974 (MNRJ).

Etimologia: O nome da espécie refere-se à localidade onde foi coletado o holótipo.

Comentário: A espécie é similar à C. lamprus Hebard, 1920 pela configuração da placa subgenital e dos estilos, diferindo dela e das demais espécies de Chromatonotus, pela configuração, no macho, das placas supra-anal, subgenital e estruturas genitais.

\section{Discussão}

As espécies de Chromatonotus assemelham-se externamente às de Ischnoptera Burmeister, 1838, contudo podem ser facilmente separadas pela ausência de modificação tergal, em forma de pente, nos sétimo e oitavo segmentos, caráter esse que é típico de Ischnoptera. A 
semelhança citada anteriormente levou Rocha e Silva Albuquerque \& Lopes (1977) a sinonimizar C. petropolitanus Rocha e Silva Albuquerque, 1971 e C. inusitatus Rocha e Silva Albuquerque, 1971, ambas as espécies do estado do Rio de Janeiro, à I. inclusa Rocha e Silva Albuquerque, 1968 e I. inusitata, respectivamente, restringindo a distribuição do gênero, no Brasil, às regiões norte e nordeste.

É assinalado em Chromatonotus, pela primeira vez, o caráter da modificação tergal evidenciada, um conjunto de cerdas simétricas no primeiro segmento abdominal que pode estar pouco nítido. Tal modificação vem corroborar o estudo desses dois gêneros

\section{Agradecimentos}

À Faperj projeto E-26/171-281/2006, Rede de Insetos - Diversidade biológica da Mata Atlântica do Estado do Rio de Janeiro pelo apoio técnico para digitalização.

\section{Referências Bibliográficas}

AMBIENTEBRASIL S/S LTDA. 2000-2008. Desenvolvimento Sustentável na Amazônia Legal http://www.ambientebrasil.com.br/composer. php3?base=./gestao/index.html\&conteudo=./gestao/artigos/amazonia. html (acessado em 20/05/2008).

GURNEY, A.B., KRAMER, J. P. \& STEYSKAL, G.C. 1964. Some techniques for the preparation, study and storage in microvials of insect genitalia. Ann. Entomol. Soc. Am. 57(2): 240-242.

EBARD, M. 1920. The Blattidae of Panamá. Mem. Am. Entomol. Soc. 4: $1-148$.

LOPES, S.M. \& OLIVEIRA, E.H. 2000. Espécie nova de Eublaberus Hebard, 1919 do Estado de Goiás, Brasil e notas sobre E. marajoara Rocha e Silva-Albuquerque, 1972 (Blaberidae, Blaberinae). Bol. Mus. Nac. N.S. Zool. 433: 1-5.

ROCHA E SILVA ALBUQUERQUE, I. \& LOPES, S.M. 1977. Blattaria (Dictyoptera) do Alto da Mosela, Petrópolis, RJ. Rev. Bras. Biol. 37(3): 499-520.

ROTH, L. M. 2003. Systematics and phylogeny of cockroaches (Dictyoptera: Blattaria). Orient. Insects 37: 1-186.

Recebido em 20/11/07 Versão Reformulada recebida 22/05/08 Publicado em 13/06/08 